\title{
Computational Intelligence in Data-Driven Modelling and Its Engineering Applications 2020
}

\author{
Qian Zhang $\mathbb{D}^{1},{ }^{1}$ Jun Chen $\mathbb{D}^{2},{ }^{2}$ and Trung Thanh Nguyen ${ }^{1}{ }^{1}$ \\ ${ }^{1}$ Liverpool John Moores University, Liverpool, UK \\ ${ }^{2}$ Queen Mary University of London, London, UK \\ Correspondence should be addressed to Qian Zhang; q.zhang@ljmu.ac.uk
}

Received 5 January 2022; Accepted 5 January 2022; Published 11 February 2022

Copyright (c) 2022 Qian Zhang et al. This is an open access article distributed under the Creative Commons Attribution License, which permits unrestricted use, distribution, and reproduction in any medium, provided the original work is properly cited.

\begin{abstract}
Modern engineering systems show increasing complexity due to their high nonlinearity and large disturbances and uncertainties introduced by them. In many cases, conventional mathematical models, such as differential equations that can accurately describe the complex systems and can be exploited in real-life applications, do not exist. However, with the fast development of advanced sensing, measurement, and data collection technologies, a large amount of data that represent input-output relationships of the systems become available. This makes data-driven modelling (DDM) possible and practical.

Data-driven modelling aims at information extraction from data and is normally used to elicit numerical predictive models with good generalization ability, which can be viewed as regression problems in mathematics. It analyses the data that characterize a system to find relationships among the system state variables (input, internal, and output variables) without taking into account explicit knowledge about physical behaviors. Many paradigms utilized in DDM have been established based on statistics and/or computational intelligence. For instance, artificial neural networks (ANNs) and fuzzy rule-based systems (FRBSs) serve as fundamental model frameworks, which are alternatives to statistical inference methods, while evolutionary algorithms (EAs), swarm intelligence (SI), and machine learning (ML) methods provide learning and optimization abilities for calibrating and improving the intelligent or statistical models. In recent years, DDM has found widespread applications, ranging across machinery, manufacturing, materials, power and energy systems, transport, and so on.
\end{abstract}

This special issue intends to bring together the state-ofthe-art research, applications, and reviews of DDM techniques. It aims at not only stimulating deep insights into computational intelligence approaches in DDM but also promoting their potential applications in complex engineering problems.

This special issue has received 27 manuscripts and 9 high-quality papers have been accepted and published (33\% acceptation rate). The accepted papers involve a variety of data-driven modelling and data analytics techniques and contribute to a wide range of application areas. A brief introduction for each contribution is provided in the following paragraphs.

O. Meza-Cruz et al. applied the techniques of ANNs and mathematical symmetry groups in modelling a thermochemical reactor of a solid-gas cooling system, where barium chloride $\left(\mathrm{BaCl}_{2}\right)$ is the solid and ammonia $\left(\mathrm{NH}_{3}\right)$ is the refrigerant. It was found that using an alternating group of mathematical symmetry in the input data of the ANN helped improve modelling precision, and using the permutations of the mathematical symmetry group in the input data helped improve the convergence speed of the training algorithm.

L. Matindife et al. designed a deep learning-based approach for a smart home application, i.e., classification of appliances, specifically for some equal or very close power specification electronic appliances (EVPSAs). They evaluated three deep learning methods for nonintrusive load monitoring (NILM) disaggregation, including the multiple parallel structures convolutional neural networks (MPS$\mathrm{CNNs})$, the recurrent neural network (RNN) with parallel dense layers for a shared input, and the hybrid convolutional 
recurrent neural network (CNN-RNN). Then, CNN and long short-term memory (LSTM) based networks were proposed for classification.

J. Liu et al. applied the ANN and neuro-fuzzy models into a sport engineering problem, which relates to the modelling the rugby players' performance under different moisture conditions. The developed intelligent models showed good performance in accuracy though using only a small number of training data. It was anticipated that the models would help the design of training programmes and the better preparation for rugby games with wet conditions.

In W. He et al.'s work, predictive models for nitrogen oxide emission were constructed and validated. In their models, CNN was employed to extract features among multidimensional data, in which the LSTM network was used to approximate the relationships among different time steps. The combination of CNN and LSTM showed better efficiency and accuracy than the baseline models. The developed models would be beneficial for providing reliable information for $\mathrm{NO}_{\mathrm{x}}$ risk assessment and management.

In H. Zhao and B. Chen's work, a complex phenomenon of rockburst was studied. A data-driven method using $\mathrm{CNN}$ was proposed to predict the potential of rockburst. The method has been compared with the conventional ANNs and shown better performance. It was assumed that such a model would help evaluate the potential of rockburst for rock underground excavation.

Y. Chen et al. tackled the problem of predicting the stock price using data-driven models. They employed the light gradient boosting machine (LightGBM) algorithm and constructed the minimum variance portfolio of mean-variance model with conditional value at risk $(\mathrm{CVaR})$ constraint. The proposed method was validated using China's stock market data between 2008 and 2018 and showed good accuracy.

C. Cheng et al. employed the data-driven models into assessing the working condition of the running gear of highspeed trains, which is complicated due to the existence of random noise in the monitoring data. Their method was developed based on a slow feature analysis-support tensor machine (SFA-STM). It was shown the developed technique could accurately anticipate the actual health status of the running gear system and outperformed the other four types of traditional data-driven models.

In the last two papers, $\mathrm{W}$. Wu et al. conducted two pieces of studies for text detection. They first proposed a pixelwise technique using instance segmentation for scene text detection. The proposed method showed good performance in the common text benchmark problems and did well in the cases including text instance with irregular shapes. In their second work, they proposed a new text detector based on weakly supervised learning. The validation results showed that the proposed method works well in scene text detection, especially for the curved texts.

\section{Conflicts of Interest}

The editors declare that there are no conflicts of interest regarding the publication of this article.

\section{Acknowledgments}

The guest editors sincerely thank all the authors for their quality contribution to this special issue. The lead guest editor would also like to express the deep gratitude to other coeditors for their great support and cooperation throughout the development of the special issue.

Qian Zhang Jun Chen

Trung Thanh Nguyen 